\title{
Calibration of TLM Model for Semiconductor Optical Amplifier by Heuristic Parameters' Extraction
}

\author{
P. Rocha ${ }^{1}$, C. M. Gallep ${ }^{2}$, T. Sutili ${ }^{1}$ and E. Conforti ${ }^{1}$, \\ ${ }^{1}$ Department of Communications, School of Electrical and Computing Engineering, University of Campinas, \\ Campinas-SP, Brazil \\ ${ }^{2}$ School of Technology, University of Campinas, Limeira-SP, Brazil
}

Corresponding Author: peterson.dmo@gmail.com

\begin{abstract}
The systemic behavior of a Semiconductor Optical Amplifier model was optimized through extensive simulations, reaching reasonable approximation to experimental obtained from commercial devices for the optical gain versus bias current, for different optical inputs powers $(-25$ up to $0 \mathrm{dBm})$, and for the gain saturation profile for different I-bias (0 up to $180 \mathrm{~mA})$. For that, parameters such as active region thickness, confinement factor, linear gain coefficient, and the transparency current were adjusted by the presented method. The method can be applied for different SOAs, enabling more accurate numerical predictions for black-box devices.
\end{abstract}

Index Terms - Semiconductor optical amplifier, calibration, extraction, TLM method.

\section{INTRODUCTION}

In recent years, the exponential increase in demand for network bandwidth keeps pushing the transmission rate growth in optical links. An attractive device to support the consequent requirement of low cost on this expansion can be the semiconductor optical amplifier (SOA), mainly regarding medium-range optical links. Several SOA applications have been proposed in linear and nonlinear regimes, such as wavelength converters [1]-[2], memory modules [3], optical buffers [4], optical space switches [5]-[8], and carrier wavelength reusing [9]-[10]. In addition, some regenerators use SOA to process optical signals, in setups with Mach-Zehnder [11] or Sagnac [12] interferometers, being able to process phase-encoded signals such as DPSK (Differential Phase-Shift Keying) [13] and QPSK (Quadrature Phase-Shift Keying) [14]. The authors have recently proposed a quasi-linear SOAbased amplifier, demonstrated in silico for 16-QAM (Quadrature Amplitude Modulation) signals with improvements for the intrinsic constellation's distortions [15].

The accurate modeling of this device can be useful to study the impact of nonlinear amplification of multilevel-encoding optical signals mentioned above. In this work, the SOA gain modeling is optimized by a calibration technique, where several intrinsic parameters are extracted heuristically. This technique is demonstrated using a commercial platform (Virtual Photonics Int, VPI [16]) that uses the Transmission Line Matrix (TLM) method to simulate the propagation of light through the 
waveguide in the SOA active region.

The SOA-model calibration was performed by matching the experimental and simulated curves of optical gain vs. current and also optical gain vs. optical input power $\left(\mathrm{P}_{\mathrm{in}}\right)$. The experimental curves were obtained from a commercial SOA (InPhenix, IPSAD1503). The SOA net optical gain was characterized by: 1) varying the bias current from 0 up to $180 \mathrm{~mA}$ and using two input optical powers, $P_{\text {in }}=-12$ and $-5 \mathrm{dBm} ; 2$ ) varying the input power from -25 up to $0 \mathrm{dBm}$ for bias currents of 40,60 , and $150 \mathrm{~mA}$.

\section{MATERIAL AND METHODS}

\section{A. Experimental Setup}

The experimental curves were obtained using the setup shown in Fig. 1: a CW laser (@1550 nm) is followed by an optical isolator to avoid back-propagation, and an OSA (Optical Spectrum Analyser) is used for optical output and noise measurements. The net optical gain is obtained by the difference between the output power and the input power $\left(\mathrm{P}_{\text {out }}-\mathrm{P}_{\mathrm{in}}\right)$, in $\mathrm{dB}$ scale. Fig. 2(a) shows the gain vs. current and Fig. 2(b) the gain vs. optical input power for the commercial SOA.

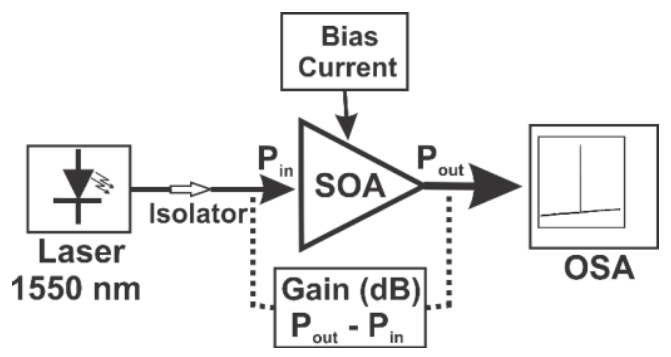

Fig. 1. Block diagram for the experimental setup.

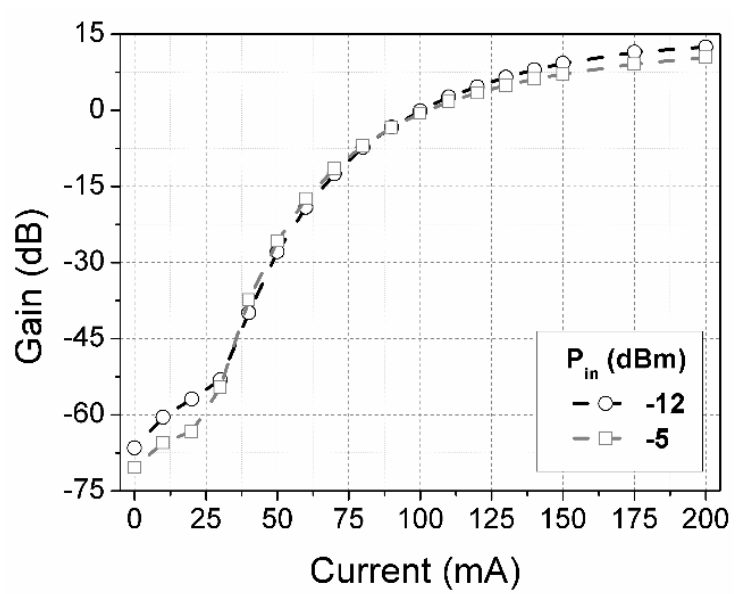

(a)

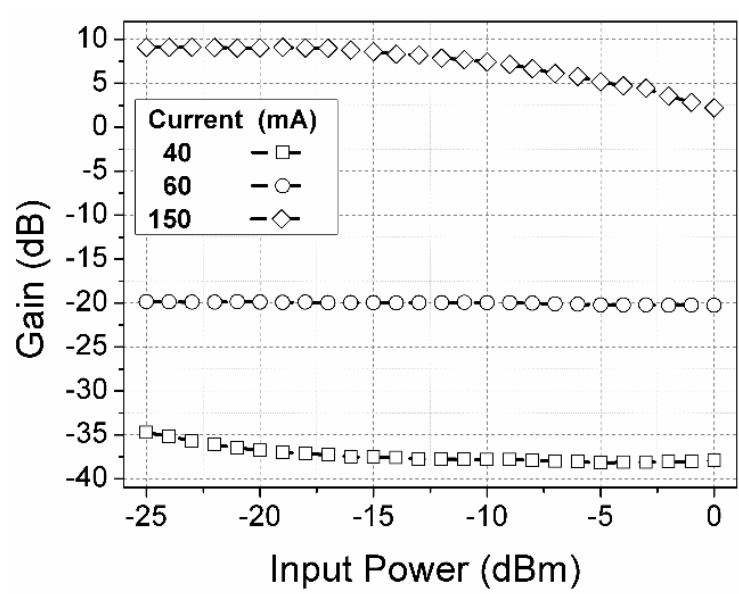

(b)

Fig. 2. SOA experimental results: (a) gain vs. bias current for input powers $\left(\mathrm{P}_{\mathrm{in}}\right)$ equal to -5 and $-12 \mathrm{dBm}$; (b) gain vs. $\mathrm{P}_{\text {in }}$ for bias currents equal to 40,60 , and $150 \mathrm{~mA}$.

The SOA active cavity's length was obtained from the distance between residual Fabry-Perot modes [17], which can be seen in the ASE spectrum for high bias current ( 200 mA). The finite facet reflectivity produces spurious longitudinal modes, and from their spacing the longitudinal length can be obtained by [18]:

Brazilian Microwave and Optoelectronics Society-SBMO received 10 Jan 2018; for review 23 Jan 2018; accepted 06 Jun 2018 Brazilian Society of Electromagnetism-SBMag (C) 2018 SBMO/SBMag 


$$
L=\frac{\lambda^{2}}{2 n_{g} \Delta \lambda} \mu m
$$

where $\lambda=1550 \mathrm{~nm}$ is the central wavelength, $n_{g}=3.86$ is the effective refractive index for the active cavity, and $\Delta \lambda=0.48 \mathrm{~nm}$ is the ripples' wavelength spacing.

\section{B. Simulations}

The TLM approach is widely used to model the light amplification inside the SOA [19]-[21]. In such approach the behavior of each TLM section must be considered as an interplay between the optical and the electronic populations [16]. Such modelling can be useful for numerical prediction on optical networks, but it requires proper calibration - otherwise non-realistic predictions appear easily. So, the device characterization must be properly designed and performed, and be equally validated for different models of commercial, black-box SOAs.

Before starting the model calibration, the SOA length must be determined, as shown above (Eq.1). After, the parameters listed in Table I are changed individually to observe and analyze their impact over the aforementioned optical gain curves. In order to do this, parameter sweep is performed for a range of values below and above a default start, preconfigured in the numerical model. These parameters are used in the rate equations and applied to the wave equations which are used to model the propagation of the optical signal within the SOA active region. The solutions of these equations are obtained numerically using the TLM method [16].

TABLE I. PARAMETERS FOR SOA MODEL - DEFAULT AND CALIBRATED

\begin{tabular}{|c|c|c|}
\hline Parameter & Default & Calibrated \\
\hline Active Region Type & MQW & MQW \\
\hline Width of Active Region & $2.5 \mu \mathrm{m}$ & $2.5 \mu \mathrm{m}$ \\
\hline Cavity Length & $650 \mu \mathrm{m}$ & $650 \mu \mathrm{m}$ \\
\hline Thickness of Active Region & $40 \mathrm{~nm}$ & $100 \mathrm{~nm}$ \\
\hline Thickness of the SCH Region & $210 \mathrm{~nm}$ & $210 \mathrm{~nm}$ \\
\hline Injection Efficiency Coefficient $\left(I_{\text {coeff }}\right)$ & 1 & 0.85 \\
\hline Internal Losses Cefficient (ILC) & $3000 \mathrm{~m}^{-1}$ & $4000 \mathrm{~m}^{-1}$ \\
\hline Internal Loss Carrier Dependence (ILCD) & $1 \cdot 10^{-23} \mathrm{~m}^{2}$ & $1.1 \cdot 10^{-21} \mathrm{~m}^{2}$ \\
\hline MQW Confinement Factor & 0.07 & 0.165 \\
\hline SCH Confinement Factor & 0.56 & 0.56 \\
\hline Optical Coupling Efficiency (OCE) & 1 & 0.4 \\
\hline Linear Gain Coefficient $\left(G_{\text {coeff }}\right)$ & $3 \cdot 10^{-20} \mathrm{~m}^{2}$ & $8.7 \cdot 10^{-20} \mathrm{~m}^{2}$ \\
\hline Carrier Density Reference Gain Shape $\left(N_{r e f}\right)$ & $2 \cdot 10^{24} \mathrm{~m}^{-3}$ & $2 \cdot 10^{24} \mathrm{~m}^{-3}$ \\
\hline Carrier Density in the Transparency $(C D T)$ & $1.5 \cdot 10^{24} \mathrm{~m}^{-3}$ & $1.7 \cdot 10^{24} \mathrm{~m}^{-3}$ \\
\hline Initial Carrier Density $(I C D)$ & $1 \cdot 10^{24} \mathrm{~m}^{-3}$ & $0.3 \cdot 10^{23} \mathrm{~m}^{-3}$ \\
\hline Nonlinear Gain Coefficient $(\varepsilon)$ & $1 \cdot 10^{-23} \mathrm{~m}^{3}$ & $1 \cdot 10^{-23} \mathrm{~m}^{3}$ \\
\hline Linear Recombination $\left(A_{\text {recomb }}\right)$ & $1 \cdot 10^{7} \mathrm{~s}^{-1}$ & $5 \cdot 10^{8} \mathrm{~s}^{-1}$ \\
\hline Bimolecular Recombination $\left(B_{\text {recomb }}\right)$ & $1 \cdot 10^{-16} \mathrm{~m}^{3} \cdot \mathrm{s}^{-1}$ & $1 \cdot 10^{-17} \mathrm{~m}^{3} \cdot \mathrm{s}^{-1}$ \\
\hline Auger recombination $\left(C_{\text {recomb }}\right)$ & $1.3 \cdot 10^{-41} \mathrm{~m}^{6} \cdot \mathrm{s}^{-1}$ & $10 \cdot 10^{-41} \mathrm{~m}^{6} \cdot \mathrm{s}^{-1}$ \\
\hline Carrier Capture Time Constant (Capture Time) & $7 \cdot 10^{-11} \mathrm{~s}$ & $1 \cdot 10^{-11} \mathrm{~s}$ \\
\hline Carrier Escape Time Constant (Escape Time) & $14 \cdot 10^{-11} \mathrm{~s}$ & $14 \cdot 10^{-11} \mathrm{~s}$ \\
\hline
\end{tabular}

First, the optical confinement factor and the cavity's transversal sizes are parameters of greatest interest. Therefore, those parameters should are the first to be tested. The confinement factor is related to the portion of the optical field within the active region and depends on the cavity thickness. The Brazilian Microwave and Optoelectronics Society-SBMO received 10 Jan 2018; for review 23 Jan 2018 ; accepted 06 Jun 2018 Brazilian Society of Electromagnetism-SBMag C 2018 SBMO/SBMag 
relationship of the three size dimensions, ie. total volume, to the confinement also determines the SOA output saturation power [22].

To analyze the mutual relationship between those, a parallel sweep was performed combining the confinement factor and the width and thickness of the active cavity and, thus, find the best match to the experimental data for the profiles of gain vs current and of gain vs $\mathrm{P}_{\text {in }}$. Figure 3 shows a case where good approximation is reached - optimized at $2.5 \mu \mathrm{m}, 100 \mathrm{~nm}$, and 0.17 , respectively. But a better adjust is still needed for the 40-80 mA range, where simulated and experimental curves differs substantially. Some preconfigured parameters may have their default value out of the actual value, and this may depend on factors such as the SOA dimensions (length, width, and thickness), the semiconductor material (InGaAsP, InGaAs, etc.), SOA structure (bulk, MQW, QD, guided index, and guided gain), etc. For that, the other parameters of Table I must be considered, such as $C D T, I C D$, $N_{\text {ref }}, G_{\text {coeff }}, \varepsilon, A_{\text {recomb }}, B_{\text {recomb }}, C_{\text {recomb }}, I_{\text {coeff }}, O C E, I L C, I L C D$, capture time and escape time, that can be varied to achieve better matchings.

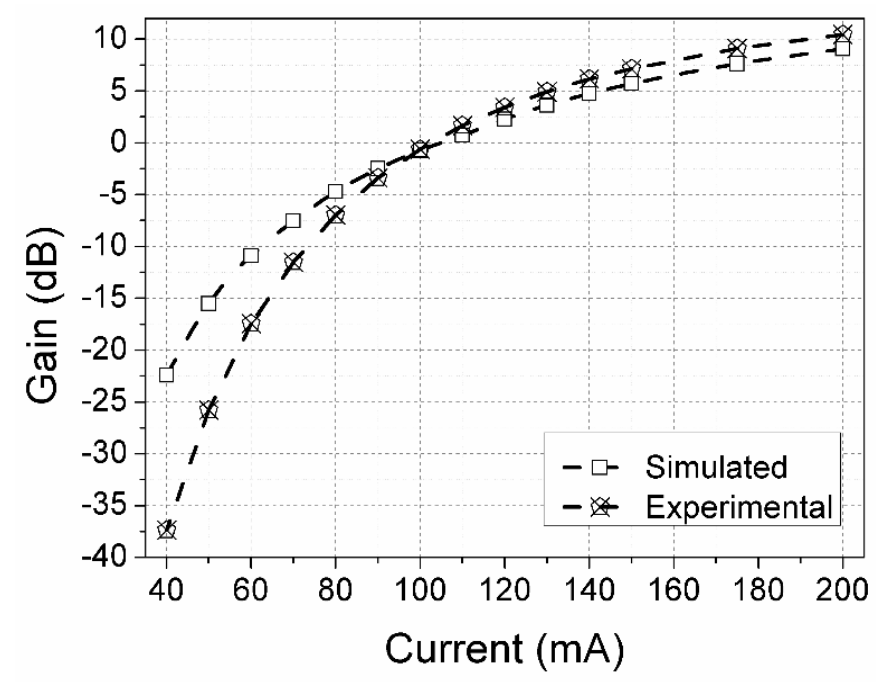

Fig. 3. Gain vs. bias current for the SOA model - length $=0.65 \mathrm{~mm}$, width $=2.5 \mu \mathrm{m}$, thickness $=100 \mathrm{~nm}$, confinement factor $=0.17$, input power $=-5 \mathrm{dBm}$.

The $C D T$ refers to the density of carriers to reach transparency, i.e. when the optical gain surpasses all losses (gain $=0 \mathrm{~dB}$ ). The $I C D$ refers to the device's intrinsic carrier's density. The amplifier optical gain is linearly dependent on the electronic carrier's density, with parabolic shaped spectra, and with effective bandwidth as function of the electronic density; the parameter $N_{\text {ref }}$ models the reference carrier density for that. The amplifier gain is also a function of the differential gain $(\mathrm{dg} / \mathrm{dN})$ [23], adjusted by $G_{\text {coeff. }}$ The parameter $\varepsilon$ contributes to the amplifier gain compression since the gain saturation occurs at very high photon density. The physical origin of this nonlinear gain coefficient is mainly the spectral hole burning [16].

The parameter $A_{\text {recomb }}$ represents the nonradiative recombination process of carriers by crystal defects (traps), that may occur in the active region during SOA fabrication and also when the device suffers aging. Carriers near those traps recombine non-radiatively, with no photon emission. This 
linear effect is known as linear recombination coefficient, being significant for low current injection [23].

The parameter $B_{\text {recomb }}$ models the interaction of two carriers, an electron in the conduction band and a hole in the valence band, meeting and recombining to produce light by spontaneous emission, whose small portion is coupled to the active waveguide [23]. The $C_{\text {recomb }}$ models the most important nonradiative recombination, the so called Auger, involving three particles that exchange energy without irradiation.

The parameter $I_{\text {coeff }}$ designates the portion of the SOA injected current reaching the active region. This current may partially deviates around the SOA electric contact, reducing so its contribution to the excited carriers' population in the conduction band. The parameter $O C E$, by its very name, represents the portion of optical power that is coupled to the amplifier coming from an optical fiber.

The parameter $I L C$ also plays an important role in the effective optical gain, so that internal losses are due to Rayleigh scattering of light, absorption of photons by material resonances, and non-uniform distribution of the optical field in the waveguide [24]. The parameter $I L C D$ changes by carriers lateral spreading and non-radiative electron-hole recombination (phonons) [19].

The capture time refers to the capture of carriers by the quantum well, after these carriers cross the $\mathrm{SCH}$ region. The escape time of carriers on the active region occurs through the thermionic emission of these carriers.

\section{RESULTS AND DISCUSSIONS}

The adjustments for the gain vs. current and gain vs. $\mathrm{P}_{\text {in }}$ profiles were done based on the model behavior, as presented in the previous section. The Fig. 4 and Fig. 5 show the experimental and numerical results after our calibration procedure.

The calibrated parameters (Table I) differ from default ones as explained: the active region was found thicker $(100 \mathrm{~nm})$ than the standard value $(40 \mathrm{~nm})$, correction based on the transparency current (see Fig. 2(a)), greater than the default set. The confinement factor differs from the standard value ( 0.07 to 0.165 ) because it is proportional to the cross-sectional area of the active region. Thus, for an increase in thickness, an increase in the confinement factor is expected. The injection efficiency coefficients $\left(I_{\text {coeff }}\right)$ models the fact that only part of the injected carriers reaches the active region, while some diffuse around the metal contact and the semiconductor, and so the calibrated value is lower than the ideal. The internal loss coefficient (ILC) of the calibrated model $\left(4000 \mathrm{~m}^{-1}\right)$ is also bigger than default $\left(3000 \mathrm{~m}^{-1}\right)$, since the internal loss is higher in thicker active regions. The fiber-towaveguide coupling is not perfect, and so the optical coupling efficiency $(O C E)$ is lower than $100 \%$, ranging from $20 \%$ to $70 \%$ [16].

The linear gain coefficient $\left(G_{\text {coeff }}\right)$ varied from $3 \times 10^{-20} \mathrm{~m}^{2}$ to $8.7 \times 10^{-20} \mathrm{~m}^{2}$ due to the strong dependence on the number of quantum wells, generally equal to or greater than 12 [25]. As mentioned above, the transparency current increases with the thickness of the amplifier, consequently the density 
of carriers in the transparency $(C D T)$ increased from $1.5 \times 10^{24} \mathrm{~m}^{-3}$ to $1.7 \times 10^{24} \mathrm{~m}^{-3}$. The initial carrier density $(I C D)$ can be used to converge the gain/current curve. Thus, the ICD value for the calibrated SOA was lower than default for convergence at low currents.

The Linear Recombination $\left(A_{\text {recomb }}\right)$ is proportional to defects during fabrication or to the SOA operation time (aging), and is significant at low currents. Thus, it was set at $5 \times 10^{8} \mathrm{~s}^{-1}$ to reduce the calibrated gain for low currents. Parameters such as capture time, $\mathrm{B}_{\text {recomb }}$ and $\mathrm{C}_{\text {recomb }}$ were used to adjust the saturation curves and the convergence of the gain/current curve. Several authors have found different values for the $\mathrm{C}_{\text {recomb }}$ as $7.5 \times 10^{-41} \mathrm{~m}^{6} \mathrm{~s}^{-1}\left(1.55 \mu \mathrm{m}\right.$ - GaInAsP) [26], 9.8x10 $0^{-41} \mathrm{~m}^{6} \mathrm{~s}^{-1}(1.65 \mu \mathrm{m}$ GaInAsP) [26], and 2.6x10 $0^{-41} \mathrm{~m}^{6} \mathrm{~s}^{-1}(1.3 \mu \mathrm{m}-$ InGaAsP) [27]. The difference between the experimental and simulated results seen in Fig. 4 (from 40 up to $80 \mathrm{~mA}$ ) and Fig. 5 (from -25 up to $8 \mathrm{dBm}$ and $60 \mathrm{~mA}$ current) shows the difficulties to adjust multi values for all model parameters.

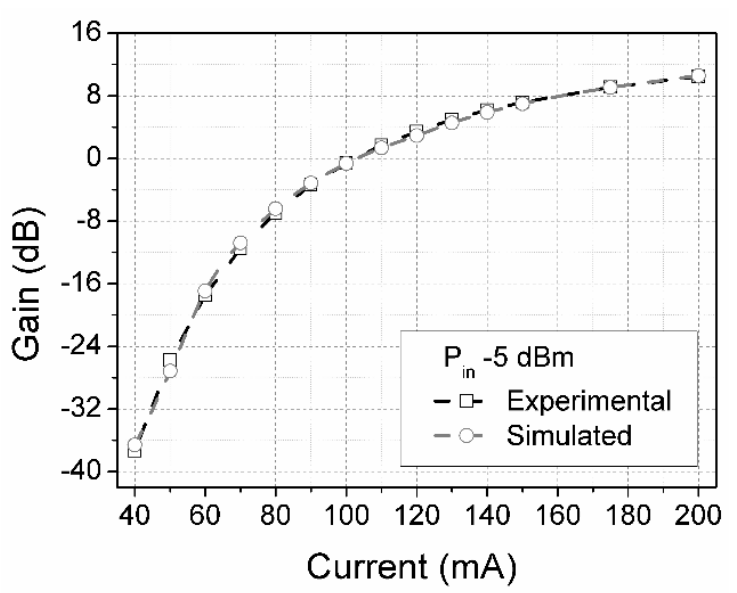

(a)

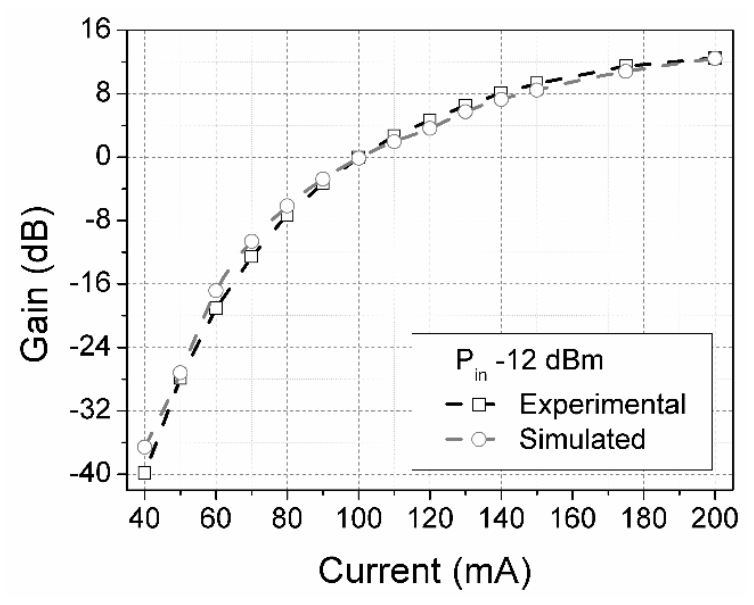

(b)

Fig. 4. Gain curve vs. bias current for the calibrated model and the experimental results: $P_{\text {in }}$ (a) $-5 \mathrm{dBm}$ (b) $-12 \mathrm{dBm}$.

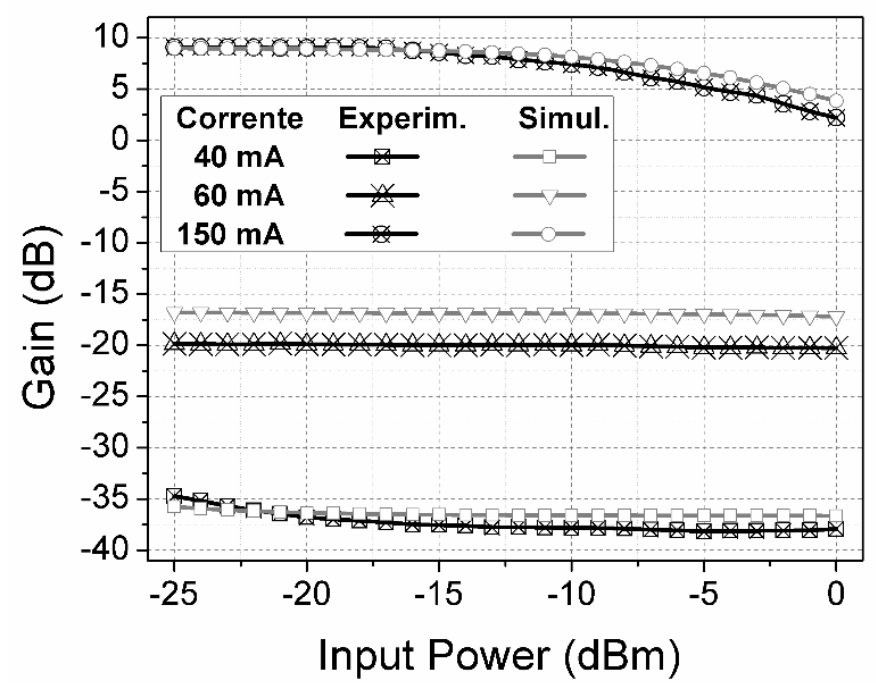

Fig. 5. Gain vs. $P_{\text {in }}$ for the calibrated model and the experimental data. 


\section{CONCLUSION}

A simple technique for calibration of numerical parameters for modelling of black-box, commercial SOAs was presented. The adjustment of the gain/current and gain/Pin curves is based on experimental data and parameters' optimization. Through this technique, it was possible to calibrate three main parameters such as thickness, width and confinement factor of the active region of a commercial SOA, among other important parameters. Thus, the calibrated model can be used in simulations to predict several types of optical sub-systems, mainly with respect to amplification and distortions caused by such devices.

\section{ACKNOWLEDGMENT}

The authors thank to the Brazilian agencies CAPES (scholarship), CNPq (contracts: 159388/2017-1, 400129/2017-5, 402923/2016-2, 301409/2017-0), and FAPESP (contracts: 2017/20121-8, 2014/18791-7, 2015/50063-4, 2007/56024-4).

\section{REFERENCES}

[1] T. Durhuus, B. Mikkelsen, C. Joergensen, S.L. Danielsen, K.E. Stubkjaer, All optical wavelength conversion by semiconductor optical amplifiers, J Lightwave Technol 14 (1992), 942-945.

[2] C. M. Gallep, A. L. R. Cavalcanti, N. S. Ribeiro, and E. Conforti, Nonhomogeneous current injection for the enhancement of semiconductor optical amplifier-based wavelength converters, Microwave Opt Technol Lett 48 (2006), 1141-1144.

[3] D. Brunina, D. Liu, and K. Bergman, An energy-efficient optically connected memory module for hybrid packet- and circuit-switched optical networks, IEEE J Sel Topics Quantum Electron 19 (2013).

[4] T. Tanemura, I. M. Soganci, T. Oyama, T. Ohyama, S. Mino, K. V. Williams, N. Calabretta, H. J. S. Dorren, and Y. Nakano, Large-Capacity Compact Optical Buffer Based on InP Integrated Phased-Array Switch and Coiled Fiber Delay Lines, J Lightwave Technol 29 (2011), 396-402.

[5] A. Ehrhardt, M. Eiselt, G. Grossopf, L. Kuller, R. Ludwig, W. Pieper, R. Schnabel, and H. G. Weber, Semiconductor laser amplifier as optical switching gate, J Lightwave Technol 11 (1993), 1287-1295.

[6] M. Renaud, M. Bachmann, and M. Erman, Semiconductor Optical Space Switches, IEEE J Sel Topics Quantum Electron, vol. 2, no. 2, Jun 1996.

[7] R. C. Figueiredo, T. Sutili, N. S. Ribeiro, C. M. Gallep, and E. Conforti, Semiconductor Optical Amplifier Space Switch With Symmetrical Thin-Film Resistive Current Injection, J Lightwave Technol 35 (2017), 280-287.

[8] R. C. Figueiredo, N. S. Ribeiro, A. M. Oliveira, C. M.Gallep, and E. Conforti, Hundred-Picoseconds Electro-Optical Switching With Semiconductor Optical Amplifiers Using Multi-Impulse Step Injection Current, J Lightwave Technol 33 (2015), 69-77.

[9] A. Chiuchiarelli, C. M. Gallep, and E. Conforti, Fabry-Perot laser-based optical switch for multicast transmission in bidirectional optical access networks, Microwave and Opt Technol Lett 58 (2016), 1466-1469.

[10] N. S. Ribeiro, C. M. Gallep, and E. Conforti, Semiconductor optical amplifier cavity length impact over data erasing/rewriting, Microwave Opt Technol Lett 55 (2013), 998-1001.

[11] L. Xi, Y. Ma, and L. Sun, Regeneration of DQPSK signals using semiconductor optical amplifier-based phase regenerator, in Int. Conf. on Advanced Infocom Technology, 2011: pp. 1.

[12] G. Gavioli and P. Bayvel, Novel 3R regenerator based on polarization switching in a semiconductor optical amplifierassisted fiber Sagnac interferometer, IEEE Photonics Technol Lett 15 (2003), 1261.

[13] P. Vorreu, A. Marculescu, J. Wang, G. Bottger, B. Sartorius, C. Bornholdt, J. Slovak, M. Schlak, C. Schmidt, S. Tsadka, W. Freude, and J. Leuthold, Cascadability and regeneration properties of SOA all-optical DPSK wavelength converters", IEEE Photonics Technol Lett 18 (2006), pp.1970-1972.

[14] Y. Zhan, Min Zhang, Mintao Liu, Lei Liu, and Xue Chen, All-Optical Signal Regeneration Based on XPM in Semiconductor Optical Amplifiers, Asia Communications and Photonics Conference, 2012.

[15] P. Rocha, C. M. Gallep, and E. Conforti, All-optical mitigation of amplitude and phase-shift drift noise in semiconductor optical amplifiers, Optical Engineering 54(10), 2015.

[16] VPItransmissionMaker, "Photonics circuits user's manual".

[17] C. M. Gallep, Redução do tempo de chaveamento eletro-óptico em amplificadores ópticos a semicondutor, $\mathrm{PhD}$ thesis in Portugueese, FEEC-UNICAMP, 2003.

[18] S. M. Sze, and K. K. Ng, Physics of Semiconductor Devices, $3^{\text {a }}$ ed., John Wiley \& Sons, New Jersey, EUA, 2007.

[19] A.J. Lowery, A new dynamic semiconductor laser model based on the transmission line modelling method, IEEE Proc J Optoelectron 134 (1987), 281-289.

Brazilian Microwave and Optoelectronics Society-SBMO received 10 Jan 2018; for review 23 Jan 2018; accepted 06 Jun 2018

$\begin{array}{lll}\text { Brazilian Society of Electromagnetism-SBMag (c) } 2018 \text { SBMO/SBMag } & \text { (cc) BY }\end{array}$ 
[20] A.J. Lowery, Transmission-line modelling of semiconductor lasers: the transmission-line laser model, Int. J. Numerical Modelling 2 (1989), 249-265.

[21] A. J. Lowery, Transmission-line laser modelling of semiconductor laser amplified communications systems, IEEE Proc J Optoelectron 139 (1992), 180-188.

[22] A. Das Barman, M. Scaffardi, S. Debnath, L Potì, and A. Bogoni, "Design tool and its experimental validation for SOA-based photonic signal processing”, Optical Fiber Techonol 15 (2009), 39-49.

[23] M. Conelly, Semiconductor Optical Amplifiers, Springer, New York, EUA, 2004.

[24] L. Gua-Dong, W. Chong-Qing, W. Fu, and M. Ya-Ya, Measurement of the internal loss coefficient of semiconductor optical amplificers, Chinese Phys Lett 30 (2013), 058501.

[25] M. C. Tatham, I. F. Lealman, C. P. Seltzer, L. D. Westbrook, and D. M. Cooper, Resonance frequency, damping, and differential gain in $1.5 \mu \mathrm{m}$ multiple quantum-well lasers, IEEE J Quantum Electron 28 (1992), 408-414.

[26] E. Wintner, and E. P. Ippen, Nonlinear carrier dynamics in $\mathrm{Ga}_{\mathrm{x}} \mathrm{In}_{1-\mathrm{x}} \mathrm{As}_{\mathrm{y}} \mathrm{P}_{1-\mathrm{y}}$ compounds, Appl Phys Lett $44 \mathrm{n}^{\circ} 2,1984$.

[27] B. Sermage, J. P. Heritage, and N. K. Dutta, Temperature dependence of carrier lifetime and Auger recombination in $1.3 \mu \mathrm{m}$ InGaAsP, Appl Phys Lett. 57, $\mathrm{n}^{\circ}$ 12, 1985. 